\title{
LOS CORALES RUGOSOS DEL CARBONÍFERO DE OSSA-MORENA: ESTADO ACTUAL DE LOS CONOCIMIENTOS
}

\author{
Sergio RODRÍGUEZ1 y Santiago FALCES ${ }^{1}$ \\ ' Departamento y U.E.I. de Paleontología, Facultad de Ciencias Geológicas e Instituto de Geología Económica. Universidad \\ Complutense, CSIC, 28040-Madrid.
}

\begin{abstract}
Rodríguez, S. y Falces, S. 1996. Los corales rugosos del Carbonífero de Ossa-Morena: estado actual de los conocimientos. [Carboniferous rugose corals from Ossa-Morena: actual stage of knowledge]. Revista Española de Paleontología, $\mathbf{N}^{\circ}$ Extraordinario, 97-102. ISSN 0213-6937.
\end{abstract}

\begin{abstract}
Rugose corals are common in the Carboniferous outcrops at Ossa Morena Zone. They occur in all marine basins containing calcareous beds. Some outcrops are already well studied and rugose corals are well known (Los Santos de Maimona, El Casar). Some others are being currently studied and some results can be now offered (Guadiato, El Pimpollar). Finally, presence of rugose corals is recorded in some outcrops, but they rest unstudied and taxa are still unknown (Santa Olalla de Cala, Pedroches). Rugose corals proved high utility for biostratigraphic, paleoecologic and biogeographic purposes in all studied areas.
\end{abstract}

Keywords: Rugosa, Spain, Ossa-Morena, Viséan, Namurian, Paleontology, Bioestratigrafía, Paleobiogeografía, Paleoecología.

\section{RESUMEN}

Los corales rugosos son frecuentes en numerosos afloramientos carboníferos dentro del ámbito de Ossa-Morena. Se encuentran en todos aquellas cuencas de origen marino que presentan niveles carbonatados. En algunas de ellas (Los Santos de Maimona, El Casar) se han estudiado ya a fondo y es posible relacionar el contenido en corales rugosos en detalle. En otras (Guadiato, El Pimpollar) se están estudiando actualmente y pueden adelantarse algunos de los taxones más frecuentes. Finalmente, en algunos afloramientos y/o cuencas (Santa Olalla de Cala, Pedroches) se conoce la presencia de corales rugosos, pero se desconocen todavía los taxones presentes. En las áreas bien estudiadas los corales rugosos han demostrado su utilidad en el reconocimiento de medios sedimentarios y en la determinación de las edades de los distintos afloramientos.

Palabras clave: Rugosos, España, Ossa Morena, Viseense, Namuriense, Paleontología, Bioestratigrafia, Paleobiogeografía, Paleoecología.

El Carbonífero de Ossa-Morena se encuentra disperso en numerosos afloramientos (Fig. 1) en general de pequeño tamaño y con estratigrafía bien diferenciada en cada caso. Salvo las cuencas de Valdeinfierno (de naturaleza continental y edad Tournaisiense) y de Belmez-Peñarroya (de naturaleza continental y edad Westfaliense) todos son de origen marino y de edad Carbonífero inferior (Quesada, 1983). En los afloramientos del Pimpollar, Santa Olalla de Cala, Los Santos de Maimona, El Casar, Campillo, Guadiato-Guadalmellato y Pedroches se localizan facies calcáreas con variado contenido paleontológico. Los corales rugosos destacan por su abundancia y variedad en algunas de ellos. El conocimiento de los mismos es, sin embargo, muy escaso en general, si exceptuamos la cuenca de Los Santos de Maimona y, en parte, la cuenca del GuadiatoGuadalmellato.

\section{AFLORAMIENTOS DEL RÍO VIAR Y SANTA OLALLA DE CALA}

La cuenca del río Viar, situada muy próxima al límite con la zona Surportuguesa, contiene materiales del Pérmico
(Simancas, 1985). Algunos niveles de calizas bioclásticas laminadas se sitúan en la zona de fractura que pone en contacto esta cuenca con materiales paleozoicos más antiguos, en la zona denominada como El Pimpollar. Originalmente fueron asignadas también al Pérmico, pero el hallazgo de corales rugosos del Carbonífero permitió reconocer que estos niveles se encontraban pinzados entre dos fracturas en el contacto entre el Pérmico y el Cámbrico. Los corales rugosos son relativamente escasos. Hasta la fecha no se han estudiado en detalle, pero a partir de muestras recogidas hace unos años se puede confirmar la presencia de Palaeosmilia murchisoni, Axophyllum sp. y Amplexocarinia sp., lo que situaría estos niveles en un Viseense superior, coincidiendo con la mayor parte de las dataciones de los niveles calcáreos de Ossa-Morena.

La cuenca de Santa Olalla de Cala presenta diversos niveles de calizas bioclásticas, frecuentemente margosas. No se han realizado investigaciones paleontológicas detalladas de dichos niveles, aunque las investigaciones sedimentológicas realizadas en esta cuenca (Santisteban Navarro et al., 1990) confirman la presencia de corales rugosos, aún no muestreados sistemáticamente ni estudiados. 


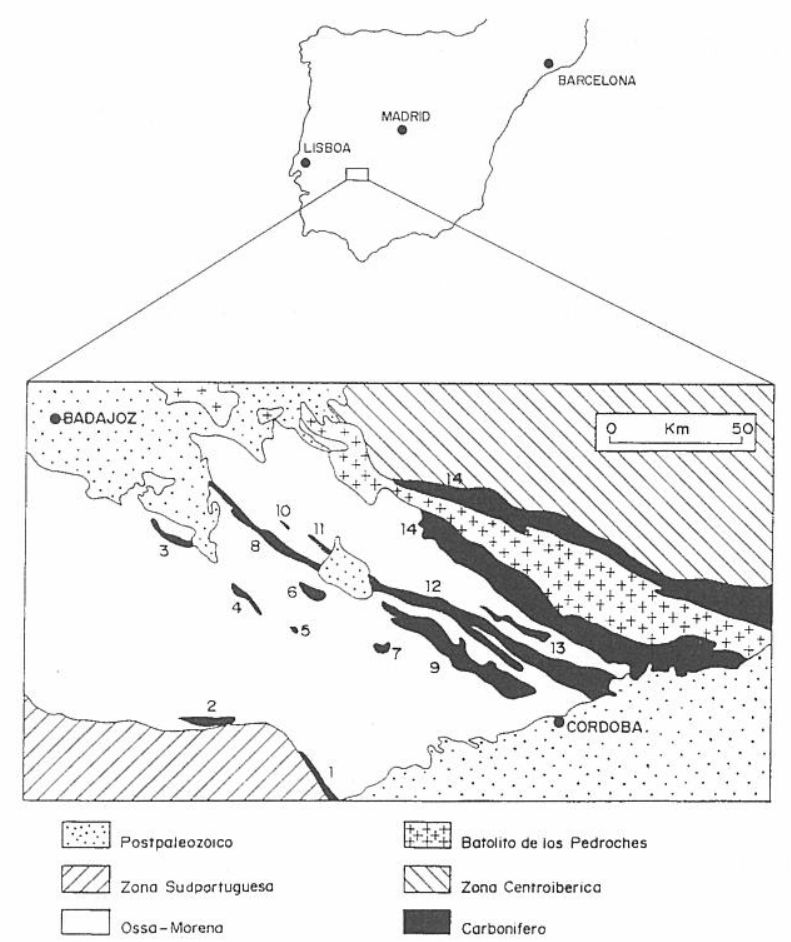

Figura 1. Afloramientos del Carbonífero en Ossa-Morena. 1.- Río Viar (El Pimpollar). 2.- Santa Olalla de Cala. 3.- Los Santos de Maimona. 4.- Bienvenida. 5.- Casas de Reina. 6.Berlanga. 7.- Valdeinfierno. 8.- Matachel. 9.- Benajarafe. 10.- El Casar. 11.- Campillo de Llerena. 12.- GuadiatoGuadalmellato. 13.- Guadalbarbo. 14.- Pedroches.

\section{CUENCA DE LOS SANTOS DE MAIMONA}

La cuenca de Los Santos de Maimona es la más profundamente estudiada tanto en los aspectos sedimentológicos como en los paleontológicos (Navarro \& Lacazette, 1922; Rosso de Luna \& Hernández-Pacheco, 1955; Altevogt, 1966; Odriozola et al., 1983; Sánchez et al., 1988,1991; Palacios González et al., 1990; Rodríguez \& Comas-Rengifo, 1989; Valenzuela et al., 1990; Soler-Gijon \& Rodríguez, 1991; Rodríguez et al., 1992, 1994 entre otros). La sucesión estratigráfica de esta cuenca ha sido dividida en ocho unidades bioestratigráficas de las que la unidades $1,3,4,5$ y 6 presentan corales rugosos (Fig. 2, Rodríguez \& Falces, 1992, 1994). Todas estas unidades han sido datadas mediante foraminíferos y corales rugosos y caracterizadas ambientalmente a partir de las características litológicas, y especialmente del contenido en algas y corales rugosos así como de otros grupos de fósiles.

En la Unidad 1, llamada informalmente "caliza de $\mathrm{Si}$ phonodendron" por la abundancia de este género de corales rugosos, y que corresponde a un complejo arrecifal (Rodríguez et al., 1992, 1994, Rodríguez \& Sánchez-Chico, 1994), predominan los corales rugosos coloniales (Siphonodendron martini, S. irregulare, Corwenia? maimonensis, Solenodendron horsfieldi), pero son también variados y frecuentes los corales solitarios con disepimentos (Dibunophyllum bipartitum, Axophyllum vaughani, A. densum, Caninia sp. Siphonophyllia cf. samsonensis, S. siblyi, Clisiophyllum aff. garwoodi).
Las unidades 3 y 4 corresponden a un sistema de barras de borde de plataforma y las facies turbidíticas calcáreas de talud asociadas. En ellas los corales rugosos, si no tan abundantes como en la caliza de Siphonodendron, sí son frecuentes e incluso más variados, ya que además de diversas especies de corales con disepimentos en los materiales correspondientes a las barras calcáreas (Axophyllum densum, Caninia sp., Siphonophyllia siblyi, Siphonodendron irregulare, S. martini, S. sociale, Siphonodendron sp., Lithostrotion araneum, $L$. vorticale, Diphyphyllum furcatum), son frecuentes los corales sin disepimentos en los niveles correspondientes a turbiditas calcáreas proximales (Amplexus sp., Zaphrentoides? sp. 1, Zaphrentites parallelus, Zaphrentites constricta, Rotiphyllum sp., Rotiphyllum? aff. densum, Zaphrentoides? aff. neogriffithi, Cyathaxonia rushiana, Sochkineophyllum sp., Ufimia sp.).

Las unidades 5 y 6 corresponden a facies de turbiditas calcáreas distales. Contienen escasos corales con disepimentos (Siphonodendron martini, Siphonodendron aff. pauciradiale, Lithostrotion decipiens), que aparecen como fragmentos claramente removilizados, pero, en cambio, abundan las formas solitarias sin disepimentos, muy bien conservadas, que con toda probabilidad habitaban los mismos medios de aguas relativamente profundas en los que se han encontrado y que no han sufrido removilización, o en todo caso muy escasa (Zaphrentites delanouei, Zaphrentites parallelus, Zaphrentites constricta, Rotiphyllum rushianum, Zaphrentoides? sp. 2, Ufimia bradbournensis, Rylstonia cf. benecompacta y Rotiphyllum aff. costatum).

La edad que han proporcionado los corales rugosos es de un Viseense superior no muy alto (Asbiense en la escala británica, Warnantiense en la escala belga) para toda la sucesión, abarcando ésta tan sólo la parte superior de la zona 6 y la inferior de la zona 7 de corales rugosos (Poty, 1985). Esta datación coincide con los datos aportados por foraminíferos (zona 15 de Mamet, 1974). Las asociaciones de corales rugosos permiten identificar una estrecha relación entre la cuenca de Los Santos de Maimona y las cuencas de la misma edad de las Islas Británicas (Mitchel, 1989) y Bélgica (Poty, 1981), con numerosas coincidencias incluso a nivel específico. La relación parece ser algo menor, pero también importante con la plataforma sahariana, donde la abundancia de axophyllidos es mayor que en las cuencas europeas (Semenoff-Tian-Chansky, 1974).

\section{AFLORAMIENTOS DE EL CASAR Y CAMPILLO DE LLERENA}

Los afloramientos de El Casar y Campillo de Llerena son pequeños retazos situados entre fallas y corresponden probablemente a fragmentos relacionados con la cuenca del Guadiato (Rodríguez, 1985). En ellos se encuentran algunos corales rugosos y tabulados en niveles calizos de edad Viseense superior (zona 15 de Mamet, 1974), situados entre conglomerados y limolitas. La asociación de corales rugosos (Palaeosmilia murchisoni, Axophyllum kirsopianum, A. pseudokirsopianum, A. tazoultense, Amygdalophylloides sp. Amplexocarinia sp.) presenta un predominio de axophyllidos, lo que la hace más semejante a las de la plataforma sahariana que las de otras cuencas de Ossa Morena. También tie- 


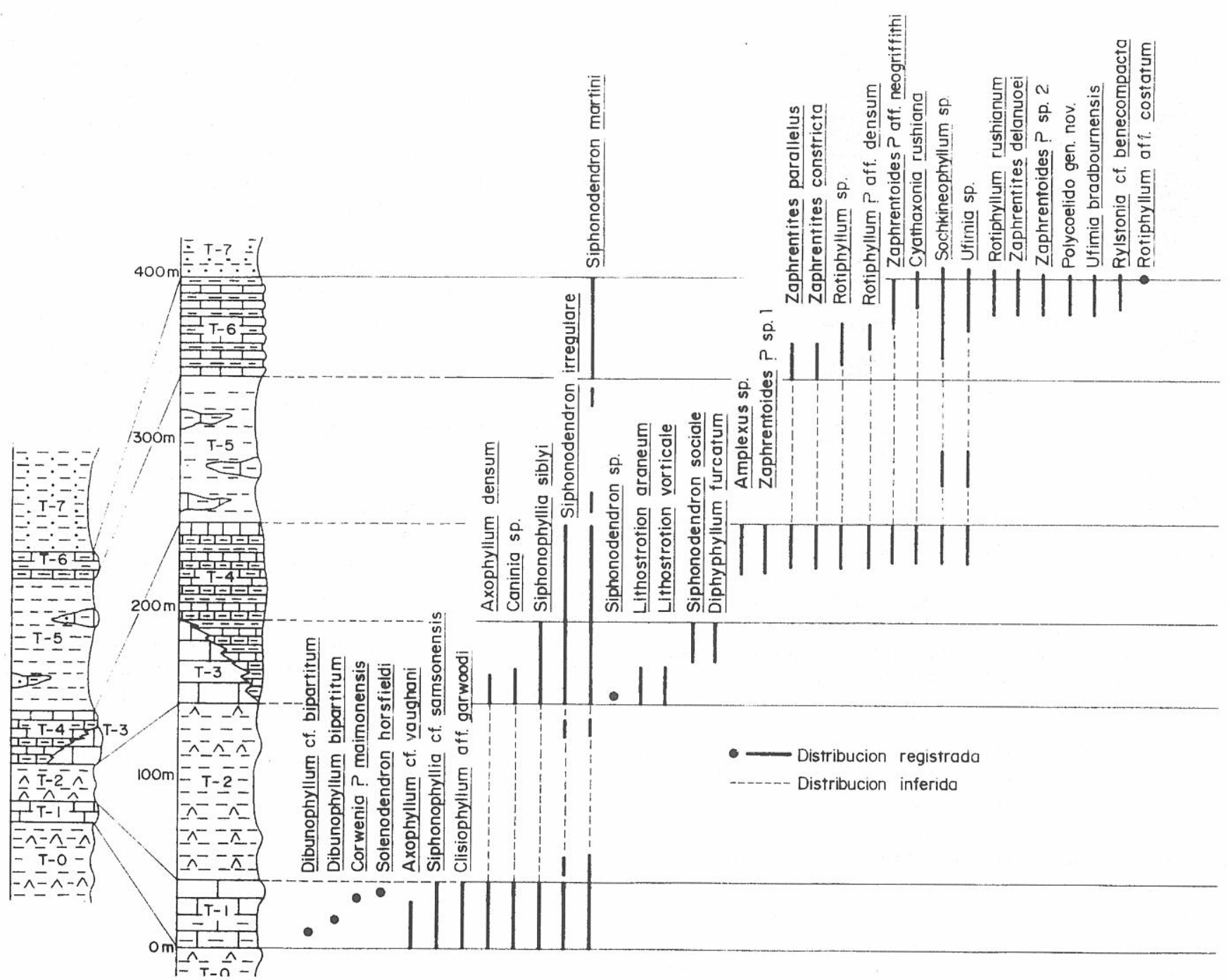

Figura 2. Columnas estratigráficas sintéticas de la cuenca de los Santos de Maimona, con la distribución de los corales rugosos. Las columnas indican las variaciones de potencia de las unidades desde el área norte (izquierda) hasta el área sur (derecha).

nen muchos taxones en común con las asociaciones descritas por Herbig (1986) en las brechas turbidíticas de la Formación Marbella en ámbito maláguide.

\section{CUENCA \\ DEL GUADIATO-GUADALMELLATO}

La cuenca del Guadiato-Guadalmellato es una de las más grandes de Ossa-Morena y de gran complejidad estructural. Hasta el momento los estudios sedimentológicos y paleontológicos son demasiado escasos para comprender en detalle esta cuenca que se prolonga más de $120 \mathrm{~km}$ en dirección NO-SE (Ortuño, 1971; Pérez-Lorente, 1979; Mamet \& Martínez, 1981, Gabaldón et al., 1983). Actualmente se está estudiando en profundidad el área comprendida entre Belmez y Villaharta. En esta zona se distinguen al menos dos conjuntos estratigráficos diferentes con facies calcáreas que presentan frecuentes corales. El primero de ellos se encuentra bien representado en la Sierra del Castillo, en las proximida- des de Espiel. Es de edad Viseense superior, y llega a incluir niveles más altos estratigráficamente que los estudiados en Los Santos de Maimona (posiblemente se alcance el límite con el Namuriense). El segundo ha sido estudiado especialmente en la zona de la Mina de San Antonio-Arroyo de la Juliana. Es probablemente de edad Namuriense inferior y su contenido en corales es menos abundante que el primero.

La sucesión de la Sierra de el Castillo se ha estudiado en tres secciones, las de la Cantera de Espiel, de el Collado y de la Sierra de la Estrella. Las dos primeras se enmarcan dentro de una misma sucesión estratigráfica y son fácilmente correlacionables entre sí (Cózar, 1994, in press). Presentan abundantes estructuras algares y frecuentes corales asociados (Fig. 3). El estudio de los corales está todavía en sus inicios, pero pudo ya identificarse gran abundancia de los géneros Siphonodendron, Lithostrotion, Palaeosmilia, Axophyllum y Siphonophyllia. Menos frecuentes parecen ser los representantes de los géneros Arachnolasma, Cionodendron, Clisiophyllum, y Dibunophyllum. También se han registrado algunos corales sin disepimentos pertenecientes a los géneros 


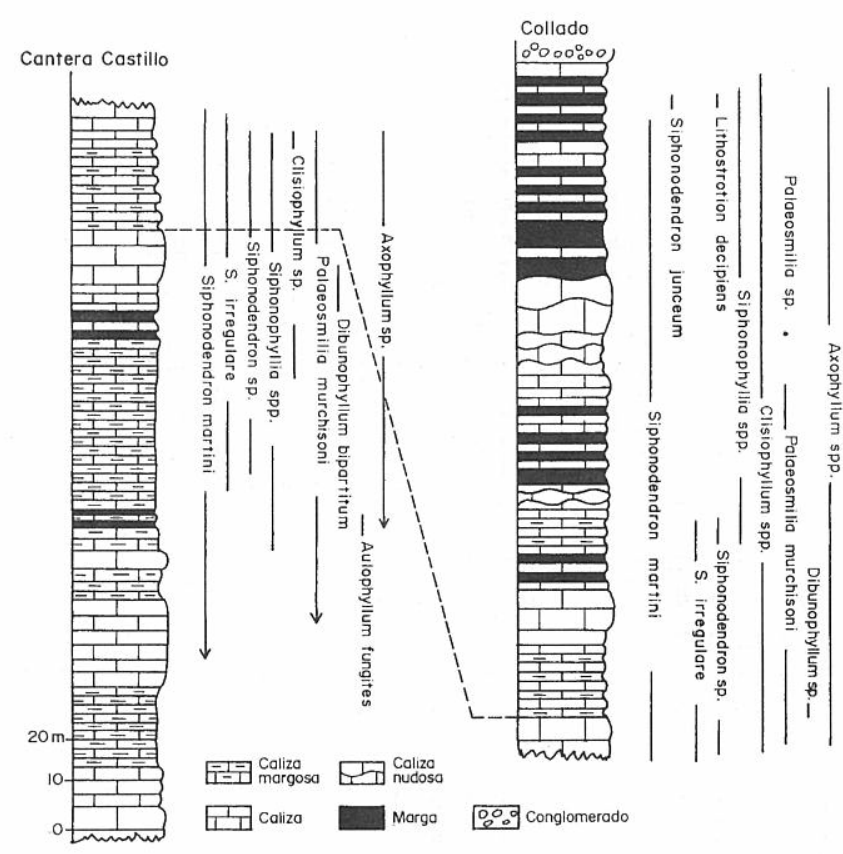

Figura 3. Columnas esquemáticas de las secciones de la cantera de Espiel y el Collado en la Sierra del Castillo (Cuenca del Guadiato), con la posible correlación entre las mismas y la distribución de los corales rugosos.

Rotiphyllum y Cyathaxonia. En el área de la Sierra de la Estrella, muy próxima a la Sierra del Castillo, se localiza una sucesión estratigráfica (Fig. 4) de difícil correlación con la anterior por causas tectónicas, que contiene escasos corales con disepimentos (algunos ejemplares de los géneros Siphonodendron, Siphonophyllia, Palaeosmilia, Axophyllum) y frecuentes corales sin disepimentos, que aparecen en diversos niveles y que constituyen algunas de las asociaciones más variadas y abundantes de la cuenca: Cyathaxonia cornu, Cyathaxonia rushiana, Cyathaxonia sp., Neaxon sp., Amplexocarinia sp., Rotiphyllum rushianum, Rotiphyllum spp., Bradiphyllum sp., Zaphrentites spp., Zaphrentoides? sp., Rylstonia spp., Sochkineophyllum sp., Ufimia bradbournensis, Pentaphyllum sp., Polycoelia sp., Amplexus sp., Cryptophyllum sp.

Las asociaciones de la Sierra del Castillo y Sierra de la Estrella permiten precisar una edad Viseense superior. La sucesión de la Sierra del Castillo corresponde a una plataforma somera, con facies que van desde una llanura intermareal hasta niveles de plataforma situados por debajo del nivel del oleaje. La sucesión de la Sierra de la Estrella por el contrario corresponde a una rampa o plataforma distal, con mayor aporte de terrígenos, aunque se reconocen en ella algunos niveles con oolitos removilizados y biohermos construidos por microorganismos y poríferos (calizas masivas en la figura 4, que corresponden a "mudstones" biolíticos.

Los materiales del arroyo de la Juliana presentan menos abundancia en corales (Ramírez Viu, 1994), que se localizan en dos facies diferentes. La primera de ellas está constituida por calizas masivas bioclásticas correspondientes a olistolitos de edad Viseense superior que contienen una asociación semejante a la mencionada en el afloramiento de El Casar (Palaeosmilia murchisoni, Siphonodendron martini, Axophyllum kirsopianum,
A. pseudokirsopianum, A. tazoultense, Amygdalophylloides sp., Ufimia bradbournensis, Amplexocarinia sp., Rotiphyllum rushianum). La segunda facies corresponde a materiales autóctonos en los que se sitúan los olistolitos. Son margas con rodolitos y niveles calcareníticos y conglomeráticos en facies canalizadas (Fig. 5), que presentan frecuentes crinoides, niveles de gigantoprodúctidos y algunos corales rugosos y tabulados. Los corales rugosos parecen indicar una edad algo más reciente, un Namuriense inferior (Aulophyllum fungites, Dibunophyllum bipartitum, Koninckophyllum sp., Palaeosmilia murchisoni, Axophyllum spp., Melanophyllum sp.).

En otros puntos de la zona norte de la cuenca del Guadiato también se han identificado asociaciones de corales rugosos. La localidad de las Caleras, en las proximidades de Peñarroya, corresponde a materiales incluidos en grandes olistolitos correlacionables con los que se localizan en el Arroyo de la Juliana, y contienen una asociación muy semejante a aquella: Palaeosmilia murchisoni, Pareynia sp., Axophyllum pseudokirsopianum, Axophyllum sp., Amygdalophylloides sp., Rotiphyllum aff. costatum, Rotiphyllum sp.

Las asociaciones de corales de la cuenca del Guadiato son semejantes a las de las cuencas británicas y belgas, aunque presentan varias peculiaridades que las aproximan también a cuencas más orientales (presencia del género Melanophyllum), a la plataforma sahariana (abundancia de axophyllidos, Semenoff-Tian-Chansky, 1974) y a la Cordillera Bética (Herbig y Mamet, 1985, Herbig, 1986).

La prolongación hacia el sudeste de esta cuenca se separa en dos bandas carboníferas con niveles calcáreos y fre-

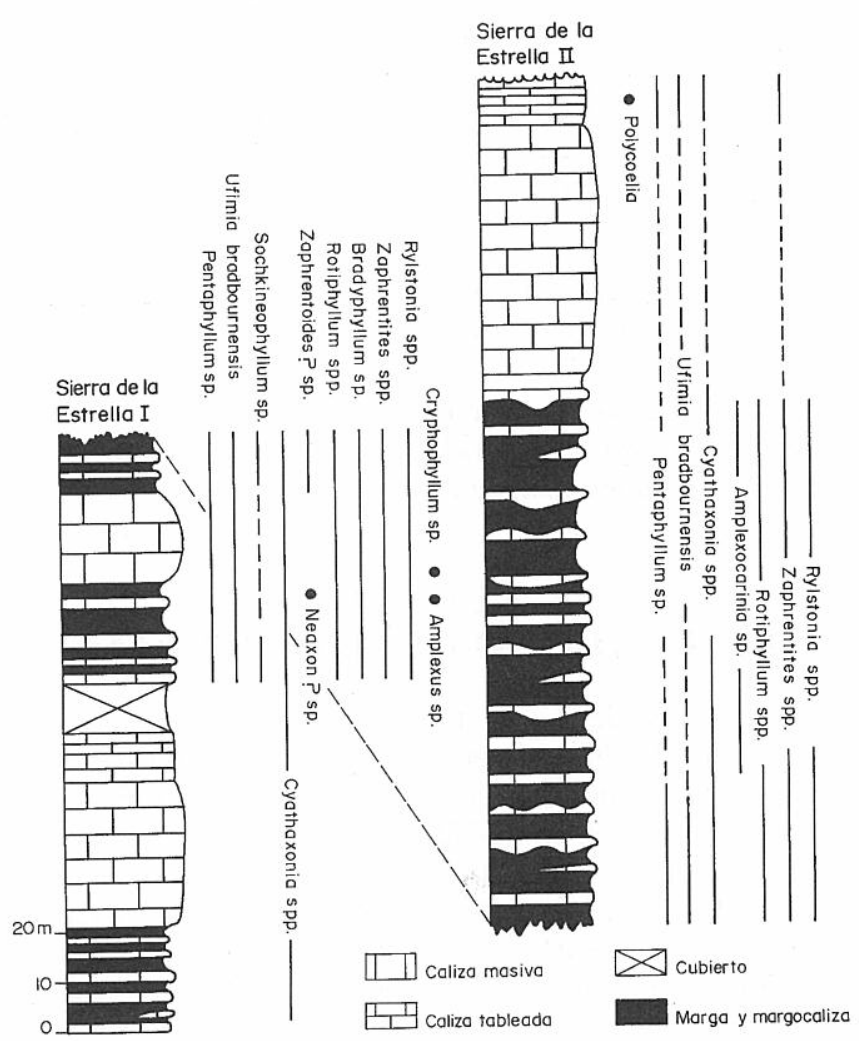

Figura 4. Columna esquemática de la Sierra de la Estrella, con la distribución de los corales rugosos. 


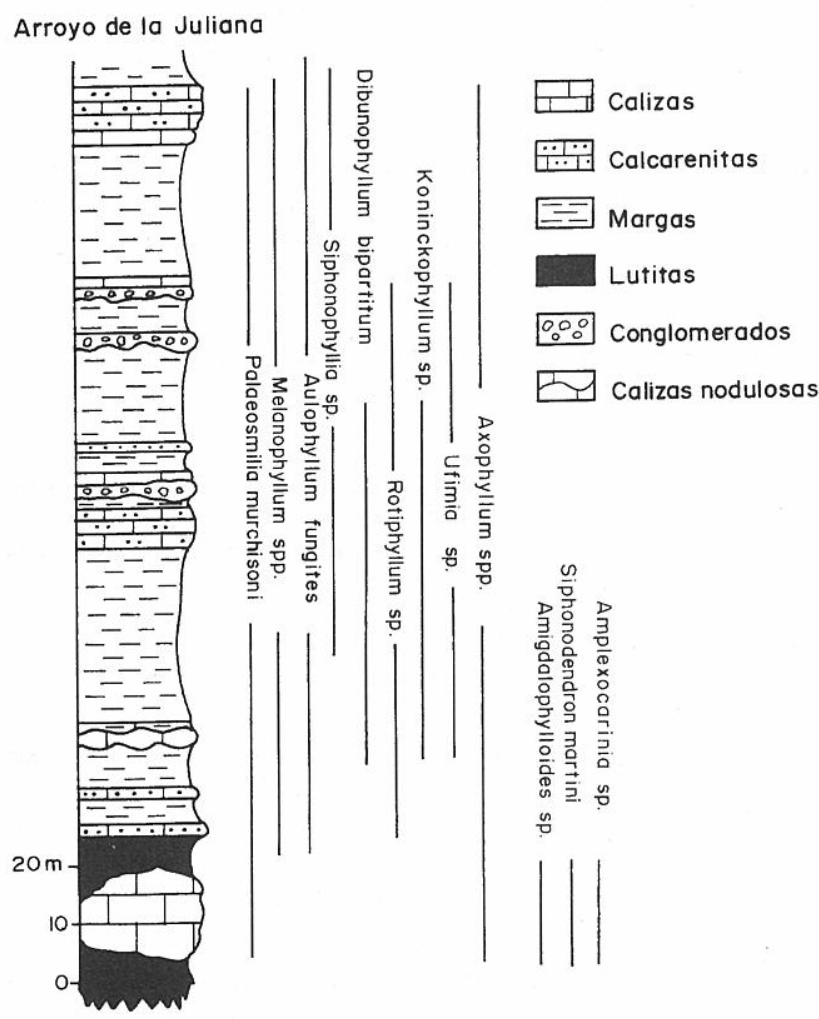

Figura 5. Columna esquemática del arroyo de la Juliana-San Antonio (Cuenca del Guadiato) con la distribución de los corales rugosos.

cuentes corales rugosos. La más oriental de ellas, que sigue aproximadamente el valle del río Guadalmellato no ha sido estudiada hasta el momento de una forma sistemática, pero se ha detectado la existencia de corales rugosos en diversos puntos. Entre estos corales destaca la presencia de Siphonodendron junceum, que define un Viseense terminal, Palaeosmilia murchisoni y Axophyllum sp.

La banda más occidental está formada por una serie de pequeños afloramientos rodeados de materiales cámbricos, que fueron someramente descritos por Liñán (1978) bajo la denominación de Cuenca Carbonífera del Santuario de Linares. La sucesión sedimentaria comienza en todos ellos con una brecha basal sobre la que se desarrolla una sucesión fundamentalmente lutítica, que en su parte inicial puede contener niveles de carbonatos con abundante contenido paleontológico. Entre estos afloramientos destacan el área carbonífera de N. ${ }^{\text {a }}$ Señora de Linares, donde Cabanas (1963) cita Cyathaxonia cornu?, los afloramientos del cerro de Los Pradillos donde se ha detectado la presencia de Palaeosmilia sp., Siphonodendron martini y Axophyllum sp., y los del cortijo de Velasco Alto, con corales semejantes a los citados.

Un poco más al este se sitúan los afloramientos de Alcolea, separados de los anteriores por materiales cámbricos y proterozoicos, y con niveles que presentan una notable bioturbación, abundantes braquiópodos y bivalvos y algunos corales rugosos coloniales de los géneros Lithostrotion y Siphonodendron.

\section{CARBONIFERO DE LOS PEDROCHES}

Finalmente, el Carbonífero de los Pedroches, que según muchos autores se sitúa en la zona Centroibérica y no en Ossa-Morena, presenta una gran extensión de afloramiento. Los materiales basales son vulcaníticos y los superiores corresponden a facies terrígenas con capas de tormentas (Gabaldón et al., 1983). Tanto en los materiales inferiores como en los superiores se intercalan lentejones de calizas bioclásticas con escasos corales de los géneros Siphonodendron y Axophyllum, siempre muy fragmentados.

\section{CONCLUSIONES}

Los corales rugosos tienen un interés indudable en el estudio de las sucesiones carboníferas de la Zona de Ossa Morena, por su utilidad en el reconocimiento de medios de sedimentación y por su uso en la bioestratigrafía de la región. Hasta el momento se conocen en detalle las asociaciones de corales rugosos de Los Santos de Maimona, que permiten establecer con precisión la edad de los distintos niveles de rocas carbonáticas presentes en esta cuenca como Viseense Superior (Asbiense) y relacionar las sucesiones de corales con las descritas en Gran Bretaña y Bélgica. Otras áreas están todavía peor estudiadas, pero los conocimientos existentes de las áreas de el Guadiato, El Casar, El Pimpollar y Pedroches permiten establecer que casi todas ellas son parte de un área de sedimentación única y que el intervalo estratigráfico representado en alguna de estas ellas es mayor que el de Los Santos de Maimona, llegando al Namuriense Inferior. Las afinidades de las asociaciones de corales rugosos en estas áreas son mayores con las descritas en la cordillera bética y la plataforma sahariana que con las de Bégica y las Islas Británicas.

\section{AGRADECIMIENTOS}

Este trabajo se incluye en el proyecto de investigación PB91-0083, subvencionado por la DGICYT. Los autores agradecemos a R. Bernal la labor de delineación y a los Dres. H. G. Herbig y L. Sánchez de Posada la revisión del manuscrito.

\section{BIBLIOGRAFÍA}

Altevogt, G. 1966. Lithostrotion scoticum (Rugosa) aus dem Visé von Zafra/Spanien. Neues Jahrbuch für Geologie und Paläontologie Monatshefte; 8, 494-500, Tübingen.

Cabanas, R. 1963. Contribución al estudio del Carbonífero de los alrededores de Córdoba. Breviora Geologica Asturica. 7(1-4), 63-67.

Cózar, P. 1994. Bioestratigrafía con foraminíferos del Carbonífero inferior de la Sierra del Castillo (Cuenca del Guadiato, Córdoba). Tesis de Licenciatura Universidad Complutense. (Inédita).

Cózar, P. (in press). Foraminíferos de la Sierra del Castillo (Banda Central de la Cuenca del Guadiato, Córdoba). Coloquios de Paleontología. 48 :

Gabaldón, V., Garrote, A. y Quesada, C. 1983. El Carbonífero inferior del Norte de la zona de Ossa Morena (SW de España). 
Compte Rendu X Congrès International de Stratigraphie et de Géologie du Carbonifère. 3, 173-186.

Herbig, H. G. 1986. Rugosa und Heterocorallia aus Obervisé-Geröllen der Marbella Formation (Betische Kordillere, Südspanien), Paläontologisches Zeitschrift, 60(3/4), 189-225.

Herbig, H. G. and Mamet, B. 1985. Stratigraphy of the limestone boulders, Marbella Formation (Betic Cordillera, Southern Spain). Compte Rendu X Congrès International de Stratigraphie et de Géologie du Carbonifère, 1, 199-212.

Liñán, E. 1978. Bioestratigrafía de la Sierra de Córdoba. Tesis Doctoral Universidad de Granada. 1-218.

Mamet, B. L. 1974. Une zonation par foraminiferes du Carbonifére inferieur de la Tethys occidentale. Compte Rendu VII Congrès International de Stratigraphie et de Géologie du Carbonifère, 3, 391-408

Mamet, B. and Martínez, C. 1981. Late Visean microfossils of the Las Caleras Bajas limestone (Córdoba, Spain). Revista Española de Micropaleontología, 13(1), 105-118

Mitchell, M. 1989. Biostratigraphy of Viséan (Dinantian) rugose coral faunas from Britain. Proceedings of the Yorkshire Geological Society, 47(3), 233-247.

Navarro, E. y Lacazette,F. 1922. Estudio de la Cuenca Carbonífera de los Santos de Maimona (Badajoz). Boletín Oficial Minero Metalúrgico, 6(63), 17-31.

Odriozola, J. M., Peón, A., Vargas, I., Garrote, A. y Arriola, A. 1983. Mapa Geológico de España a escala 1:50.000. Hoja 854; Zafra. I.G.M.E., segunda edición, Madrid.

Ortuño, G. 1971.- Middle Westphalian strata in South-West Spain. Compte Rendu VI Congrès International de Stratigraphie et de Géologie du Carbonifère, 3, 1275-1279.

Palacios González, M. J., Palacios, T. y Gómez Valenzuela, J. M. 1990. Trilobites y Goniatites de la Cuenca Carbonífera de Los Santos de Maimona, deducciones bioestratigráficas. Geogaceta, 8, 66-67.

Pérez-Lorente, F. 1979. Geología de la zona Ossa-Morena al norte de Córdoba (Pozoblanco-Belmez-Villaviciosa de Córdoba). Tesis Doctoral Universidad de Granada, 281, 1-340.

Poty, E. 1981. Recherches sur les tétracoralliaires et les hétérocoralliaires du Viséen de la Belgique. Mededelingen Rijks Geologische Dienst, 35, 1-161.

Poty, E. 1985. A rugose coral biozonation for the Dinantian of Belgium as a basis for a coral biozonation of the Dinantian of Eurasia.- Compte Rendu X Congrès International de Stratigraphie et de Géologie du Carbonifère, 4, 29-31.

Quesada, C. 1983. El Carbonífero de Sierra Morena. In: C. Martínez Díaz (Ed.). Carbonífero y Pérmico de España, 243-278. Ministerio de Industria y Energía.

Ramírez-Viu, F. 1994. Estudio de los corales rugosos del Carbonifero del sector del Arroyo de la Juliana (Cuenca del Guadiato, Córdoba). Tesis de Licenciatura Universidad Complutense. (Inédita).

Rodríguez, S. 1985. Aportaciones de los Corales Rugosos a la datación del Devónico y Carbonífero de Ossa Morena. In: $5^{a}$ Reunión del Grupo de Ossa Morena, Temas Geológico Mineros: 7, 2-6 Instituto Geológico y Minero de España.

Rodríguez, S., Arribas, M. E., Comas-Rengifo, M. J., de la Peña, J. A., Falces, S., Gegúndez, P., Kullman, J. Legrand-Blain, M., Martínez-Chacón, M. L., Moreno-Eiris, E., Perejón, A., Sán- chez, J. L., Sánchez-Chico, F. y Sarmiento, G. 1992. Análisis Paleontológico y Sedimentológico de la cuenca carbonífera de Los Santos de Maimona (Badajoz). Coloquios de Paleontología. 44, 1-232.

Rodríguez, S., Arribas, M. E., Falces, S., Moreno-Eiris, E. and De la Peña, J. 1994. The Siphonodendron Limestone of Los Santos de Maimona Basin: development of an extensive reef-flat during the Visean in Ossa Morena, SW Spain. Courier Forschungsinstitut Senckenberg, 172, 203-214.

Rodríguez, S. y Comas-Rengifo, M. J. 1989. Los Heterocorales del Carbonífero de los Santos de Maimona (Badajoz, SW de España). Coloquios de Paleontología, 42, 61-81. Editorial Universidad Complutense. Madrid.

Rodríguez, S. y Falces, S. 1992. Corales Rugosos. In: Rodríguez, S., Ed., Análisis paleontológico y sedimentológico de la cuenca carbonífera de Los Santos de Maimona (Badajoz). Coloquios de Paleontología, 44, 159-218.

Rodríguez, S. and Falces, S. 1994. Coral distribution patterns at "Los Santos de Maimona" Lower Carboniferous basin (Badajoz, SW Spain). Courier ForschungsInstitut Senckenberg, 172, 193-202.

Rodríguez, S. y Sánchez-Chico, F. 1994. Bioconstrucciones de corales rugosos y algas calcáreas de la sección del Torreón (Viseense, Badajoz). Coloquios de Paleontología, 46, 59-73

Rosso De Luna, I. y Hernández Pacheco, E. 1955. Mapa Geológico de España a escala 1:50.000. Explicación de la hoja $n^{\circ} 854$ (Zafra). Instituto Geológico y Minero de España, 1-142 Madrid.

Sánchez, J. L., Comas-Rengifo, M. J. y Rodríguez, S. 1988. Estudio estratigráfico de los materiales carbonatados del Carbonífero de los Santos de Maimona (Badajoz, SO de España). Comunicación en el segundo Congreso de Geología de España, 1, 197-200.

Sánchez, J. L., Comas-Rengifo, M. J. y Rodríguez, S. 1991. Foraminíferos del Carbonífero inferior de los Santos de Maimona (Badajoz, SO de España). Boletín de la Real Sociedad Española de Historia Natural (sección de Geología), 86(1-4), 101147.

Santisteban-Navarro, J. I., Alonso-Gavilán, G. y Dabrio, C. J. 1990. Sedimentación de plataforma durante el Carbonífero inferior (Formación Terena) en el área de Santa Olalla de Cala (Huelva, España). Boletín de la Real Sociedad Española de Historia Natural, 85(1-4), 83-100

Semenoff-Tian-Chansky, P. 1974. Recherches sur les Tétracoralliaires du Carbonifère du Sahara occidental. Centre des recherches sur les zones arides Ser. Géol. 21:1-316.

Simancas, J. F. 1985. Estudio estratigráfico de la cuenca del Viar. Temas Geológico-mineros, 7, 7-17.

Soler Gijón, R. y Rodríguez, S. 1991. Estudio de un resto de Bradiodonto (clase Chondrictyes) del Viseense de los Santos de Maimona (Badajoz, SO de España). Coloquios de Paleontología, 43, 101-114.

Valenzuela, J. M., Palacios, T. y Palacios-González, M. J. 1990. Aspectos paleoecológicos de la cuenca de los Santos de Maimona: acritarcos y esporas. Geogaceta, 8, 118-121.

Manuscrito recibido: 5 de noviembre, 1994 Manuscrito aceptado: 15 de junio, 1995 